\title{
Bimetallic Nanomaterials: Functional Efficacy towards Synthesis, Photocatalytic Degradation, and Biomedical Catalysts
}

\author{
S. Mohana Roopan, ${ }^{1}$ Amir Kajbafvala, ${ }^{2}$ Arash Dehghan Banadaki, ${ }^{2}$ \\ G. Madhumitha, ${ }^{1}$ and Karthik S. Pillai ${ }^{3}$ \\ ${ }^{1}$ School of Advanced Sciences, VIT University, Vellore, Tamil Nadu 632014, India \\ ${ }^{2}$ Department of Materials Science and Engineering, North Carolina State University, 911 Partners Way, \\ Engineering Building I, Raleigh, NC 27695-7907, USA \\ ${ }^{3}$ Technology Development Division, Globalfoundries Inc., Malta, NY 12020, USA
}

Correspondence should be addressed to Amir Kajbafvala; akajbaf@ncsu.edu

Received 8 May 2014; Accepted 8 May 2014; Published 12 August 2014

Copyright (C) 2014 S. Mohana Roopan et al. This is an open access article distributed under the Creative Commons Attribution License, which permits unrestricted use, distribution, and reproduction in any medium, provided the original work is properly cited.

Considerable efforts have been dutiful to bimetallic nanomaterials with various architectures due to their interest from both scientific and technological outlooks for the modification of physical and chemical properties of metal nanomaterials. Bimetallic colloids function in favor of environment and biomedical catalysis, but they are distinct from those of the corresponding monometallic nanomaterials. Many methods for the preparation of bimetallic nanomaterials have been reported, such as alcohol and citrate reduction, hydrothermal, sonochemical method, coprecipitation, reverse micelles, and biosynthesis. Among all, the biosynthesis of metal nanomaterials has been explored as an alternative to developing environmentally benign procedures. Alternatives for the synthesis of bimetallic nanomaterials are currently being explored through bacteria, plant biomass, live plants, and their extracts. The catalytic property of metal nanomaterials is also of great interest because of their particle size.

This special issue presents the recent advances and techniques for producing and evaluating the mechanical and catalytic properties of bimetallic nanomaterials. Due to the issue's focus towards mechanistic studies, photocatalytic degradation, biomedicine, and catalytic application in organic reactions, we were particularly interested in articles describing novel bimetallic nanomaterials' design and application. It contains six articles including two reviews and four research articles.

In their paper, "Analysis of $\mathrm{Pd}-\mathrm{Ni}$ nanobelts melting process using molecular dynamics simulation," C. Gang et al. simulated the heating process of Pd-Ni alloy nanobelts using molecular dynamics (MD) and calculated the melting temperature of these features with the aid of thermodynamic concepts. The melting and breaking of $\mathrm{Pd}$ $\mathrm{Ni}$ alloy nanobelts can be indicated by increasing and decreasing regions of total energy relating to temperature curve. With increasing temperature, number of face center cubic (FCC) pairs will decrease and almost disappear at melting point. The melting point is lower than bulk phase $\mathrm{Pd}-\mathrm{Ni}$ alloy, and the melting point obtained by $\mathrm{MD}$ is close to the one calculated by thermodynamic method.

In their research article "Sol-gel synthesized magnetic $\mathrm{MnFe}_{2} \mathrm{O}_{4}$ spinel ferrite nanoparticles as novel catalyst for oxidative degradation of methyl orange," L. Zhang and Y. $\mathrm{Wu}$ prepared the $\mathrm{MnFe}_{2} \mathrm{O}_{4}$ spinel ferrite (MNF) nanoparticles from metal nitrates by the sol-gel process followed by calcination at different temperatures. Compared to the other MNF nanoparticles, the MNF-500 nanoparticles had a single crystalline phase; their specific surface area was 
$50.2 \mathrm{~m}^{2} \cdot \mathrm{g}^{-1}$ and had better low-temperature oxidation activity. Due to the large number of active oxygen species on the surfaces and the ion transference of $\mathrm{Mn}$ and Fe elements, the MNF-500 nanoparticles showed a high methyl orange degradation efficiency up to $98 \%$. Additionally, the saturation magnetization of the MNF-500 nanoparticles was $43.1 \mathrm{~A} \cdot \mathrm{m}^{2} \cdot \mathrm{kg}^{-1}$ that made them easy to separate from the methyl orange solution by an external magnetic field. Thus, this kind of $\mathrm{MnFe}_{2} \mathrm{O}_{4}$ nanoparticles will have a potential for oxidative degradation of dye in the water treatment fields.

In their paper, "Ag-Cu colloid synthesis: bimetallic nanoparticle characterisation and thermal treatment," J. Sopoušek et al. prepared Ag-Cu bimetallic colloidal nanoparticles (NPs) by solvothermal synthesis from metalloorganic precursors in a mixture of organic solvents. The nanoparticles were characterized by dynamic light scattering (DLS) and small angle X-ray scattering (SAXS). The properties of metallic core and organic shell of the nanoparticles were studied by direct inlet probe mass spectrometry (DIP/MS), Knudsen effusion mass spectrometry (KEMS), double-pulse laserinduced breakdown spectroscopy (DPLIBS), and differential scanning calorimetry (DSC). The transmission electron microscopy (TEM) and scanning electron microscopy (SEM) were also used for particle characterization before and after thermal analysis. Their experiment yielded results that were for $\mathrm{Ag}-\mathrm{Cu}$ nanoparticles for the first time. The detected liquidus temperature has been compared with the prediction obtained from the calculation of the phase diagram of Ag-Cu nanoalloy. The experimental results also showed that of the near-eutectic composition, $\mathrm{Ag}$ - $\mathrm{Cu}$ nanoparticles possess the FCC crystal lattice. Surprisingly, spinodal decomposition was not observed inside the $\mathrm{Ag}$ - $\mathrm{Cu}$ nanoparticles at temperatures up to $230^{\circ} \mathrm{C}$. The eutectic Ag-Cu microparticles are formed before melting.

In "Preparation and characterization of $A u / P d$ modified$\mathrm{TiO}_{2}$ photocatalysts for phenol and toluene degradation under visible light - the effect of calcination temperature," A. Cybula et al. reported the effect of calcination temperature on photocatalytic activity under visible light in toluene and phenol degradation for rutile modified with $\mathrm{Au} / \mathrm{Pd}$ monometallic and bimetallic nanoparticles. The sample that contains $0.5 \mathrm{~mol} \% \mathrm{Pd} / \mathrm{TiO}_{2}$ exhibited the highest activity under visible light irradiation in gas and aqueous phase reaction among all photocatalysts calcined at $350^{\circ} \mathrm{C}$, while the sample modified only with gold nanoparticles showed the lowest activity. The $\mathrm{Au} / \mathrm{Pd}-\mathrm{TiO}_{2}$ sample calcinated at $350^{\circ} \mathrm{C}$ possesses the highest photocatalytic activity when degrading phenol under visible light, which is more than 14 times higher than that of the sample calcinated at $450^{\circ} \mathrm{C}$. Enhancement in annealing temperature to $450^{\circ} \mathrm{C}$ resulted in an increase of palladium concentration in the outer shell for small nanoparticles and in the drop of photoactivity in phenol and toluene degradation under visible light. Further increase of temperature to $700^{\circ} \mathrm{C}$ caused a slight increase in activity in the gas phase and no change in photoactivity in the aqueous phase under visible light irradiation, probably due to the formation of homogenous alloys structures.
In their review, "Recent advances in facile synthesis of bimetallic nanostructures: an overview," A. D. Banadaki and A. Kajbafvala illustrate that the synthesis of bimetallic nanocrystals with controlled morphologies has attracted much attention during the last decade based on their efficacy in various applications particularly in catalysis, sensing, and drug delivery. Among several methods, wet-chemical (colloidal) synthesis methods have been more popular due to their ease of use and their potential for implementing environmentally friendly production routes. They categorized these methods in four groups, namely, continuous growth, crystalline coalescence, core/shell growth, and galvanic replacement. Based on the type of application, each of the above may be preferred; however, galvanic replacement and core/shell growth methods are the two most preferred synthesis methods in the recent years. It has been observed that the morphology of the product is dependent on many factors, most importantly temperature of the reaction, reaction duration, facet bias of capping agents, and reduction potential of any of the involved agents. Despite recent advances in controlling the morphologies of nanostructures, the literature suggests that only the morphology of the $\mathrm{Pt}$ and Pd based nanocrystals can readily be determined while the similar control is not evident for other noble metals. Recent literature in this field suggests that researchers are employing organic and less powerful reductants to reduce the environmental impact associated with the production of these materials. However, use of such materials inhibits the use of nonnoble metals that are readily available with less cost. In the end they conclude that, despite the performance of the products (multiple times better than the parent metals) and the advances in producing them, many of the proposed methods are only applicable in laboratory scale and are still expensive to be implemented in the industrial scale.

In her review, "Progress, challenge, and perspective of bimetallic $\mathrm{TiO}_{2}$-based photocatalysts," A. Zielińska-Jurek mentioned that bimetallic photocatalysts have attracted considerable attention in recent years as a class of highly active catalysts and photocatalysts active under both UV-Vis and light irradiation. Bimetallic alloy nanoparticles possess the ability to absorb visible light, in a wide wavelength range (broad LSPR peak), and therefore revealed the highest level of activity as a result of utilization of a large amount of incident photons. On the other hand, bimetallic nanoparticles can enhance the rate of trapping photo-excited electrons and inhibit the recombination process due to the capability of the storage of photo-excited electrons. Based on the literature, two groups of the bimetallic photocatalysts were distinguished. The first group includes bimetallic $\mathrm{TiO}_{2}$ photocatalysts (BMOX) highly active under UVVis and light irradiation in a variety of oxidation reactions, and the second type presents bimetallic photocatalysts (BMRED) exceptionally active in hydrogenation reactions. This review summarized recent advances in the preparation and environmental application of bimetallic photocatalysts. Moreover, the effects of various parameters such as particles shape, size, amount of metals, and calcination on the photocatalytic activity of bimetallic photocatalysts are also discussed. 


\section{Acknowledgments}

We want to express our sincere appreciation to all the authors for their contribution. Moreover, our thanks are extended to all the reviewers for their time and for enhancing the quality of these articles. S. Mohana Roopan and Amir Kajbafvala contributed equally to this special issue.

S. Mohana Roopan Amir Kajbafvala Arash Dehghan Banadaki G. Madhumitha

Karthik S. Pillai 

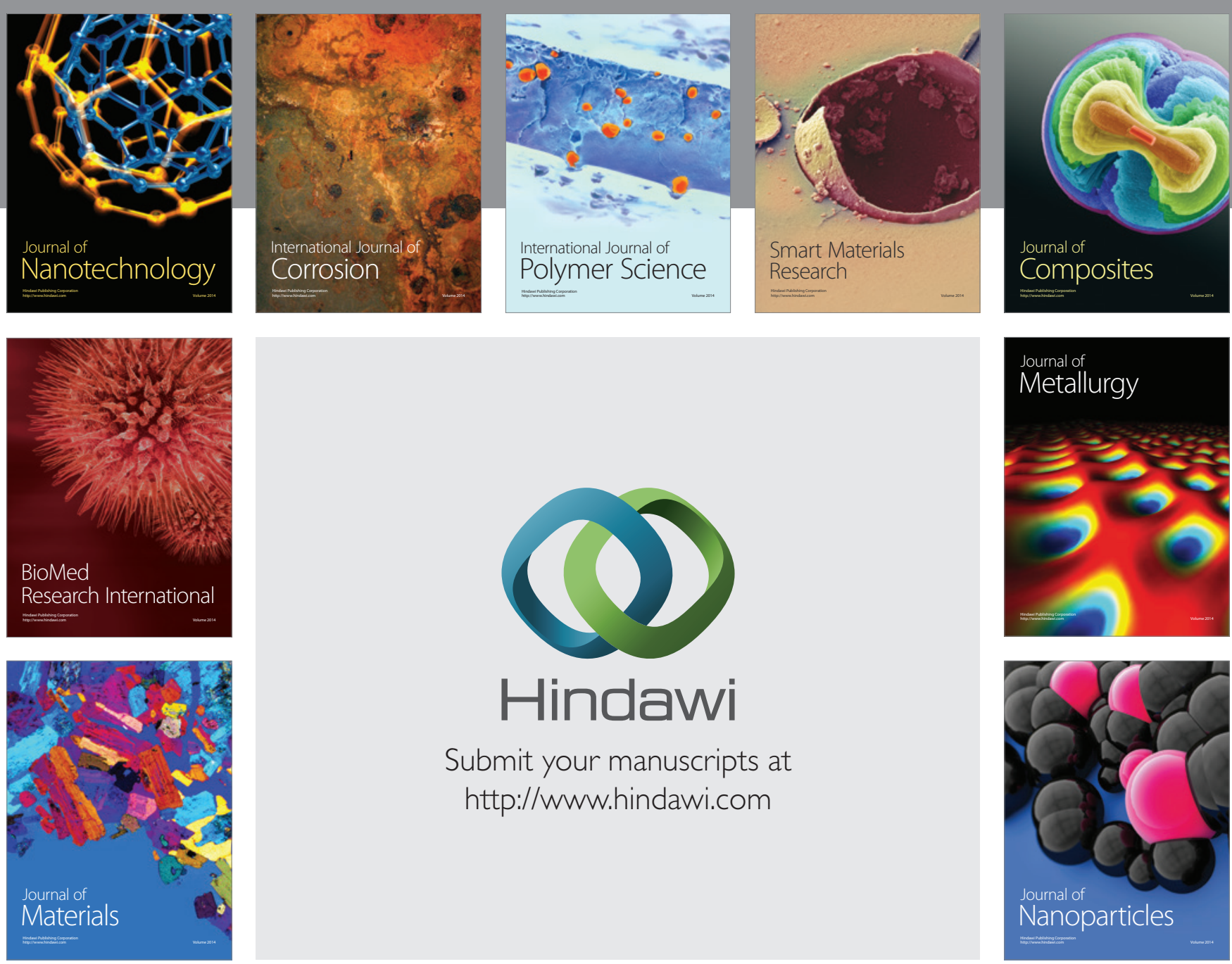

Submit your manuscripts at http://www.hindawi.com
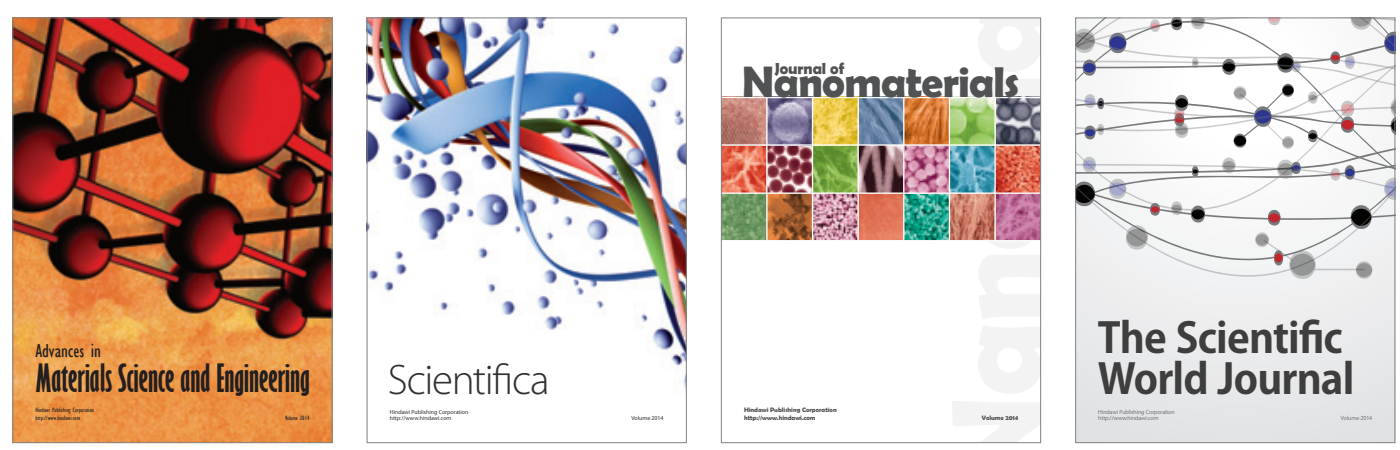

\section{The Scientific World Journal}
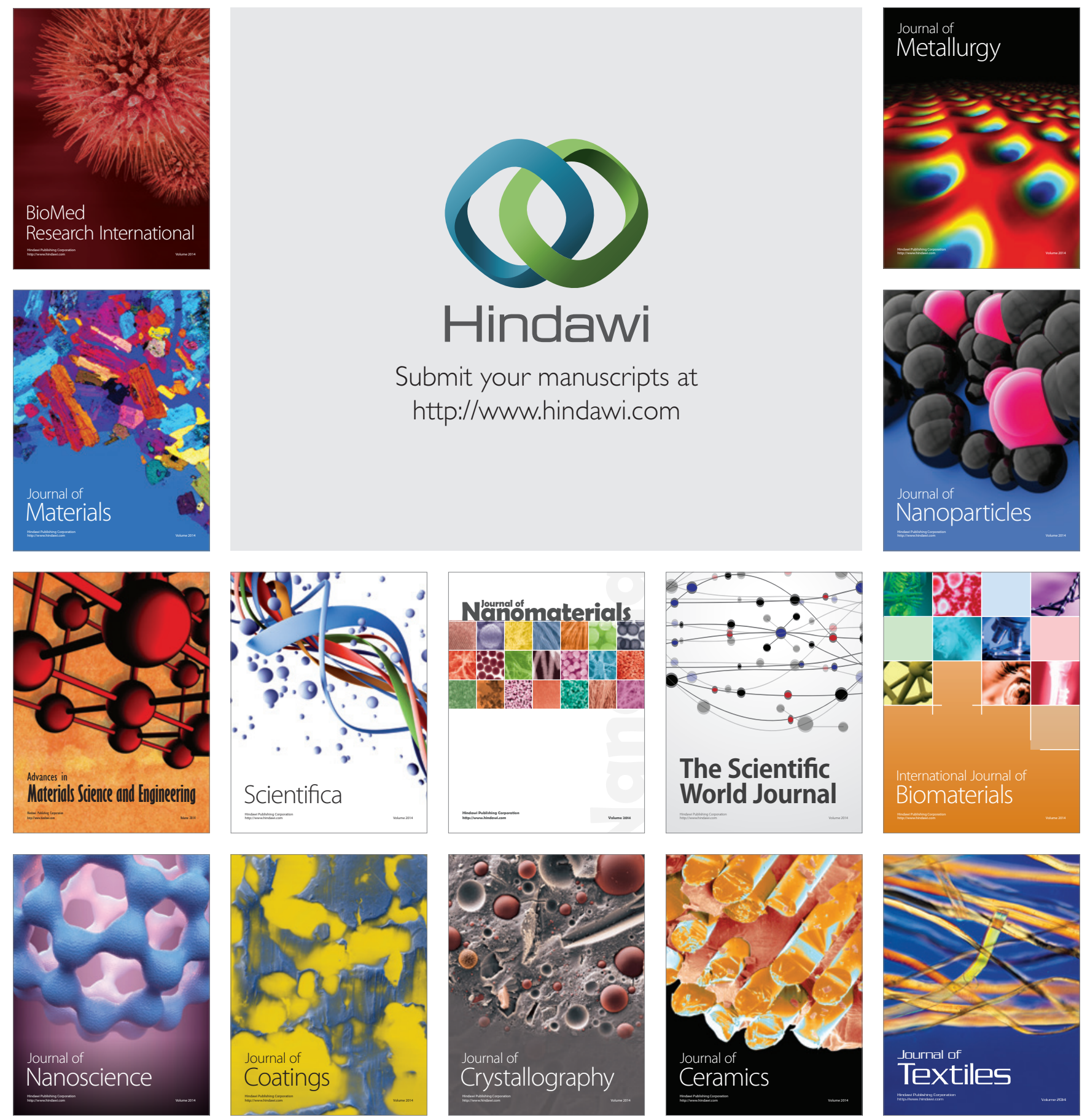